\title{
Amplitude of Care for the Neonatal Brain
}

\author{
SReenath Thati Ganganna, ${ }^{\mathbf{1}}$ Rawad Obeid ${ }^{2}$ \\ ${ }^{1}$ Division of Child Neurology, University of Iowa, Stead Family Children's Hospital, Iowa City, IA, USA; \\ ${ }^{2}$ Beaumont Children's Hospital and Oakland University William Beaumont School of Medicine, Royal Oak MI, USA. \\ Correspondence to: Sreenath Thati Ganganna, Clinical Assistant Professor, Clinical Director, Interim Division Director, \\ Division of Child Neurology, University of Iowa, Stead Family Children's Hospital, 200 Hawkins Drive, Iowa City, IA 52242, USA. \\ sreenath-thatiganganna@uiowa.edu
}

$\mathrm{N}$ eonatal encephalopathy is still a significant problem worldwide with an incidence estimated at 3 per 1000 live births [1]. It is a clinical condition encountered in the very early days of life of a newborn, characterized by depressed level of consciousness and/or seizures, often associated with abnormal tone and reflexes, and autonomic dysfunction [2]. This condition can be secondary to a plethora of etiologies during prenatal, intrapartum to postnatal period that can lead to cerebral injury [3]. There can be a variety of adverse short term and long term neurodevelopmental outcomes of neonatal encephalopathy, based on the etiology and severity of injury. In the last couple of decades, there has been a growing interest in developing accurate physiological, radiological and biochemical markers for a reliable prediction of outcome in patients with neonatal encephalopathy [4].

Amplitude-integrated EEG (aEEG) is one such biomarker that is widely used in neonatal intensive care unit (NICU) as a simple clinical tool to assess neonatal encephalopathy as it provides a real time and continuous monitoring of the cerebral activity. In addition to its use in detecting neonatal seizures, it is also helpful in predicting neurodevelopmental prognosis. aEEG represents a processed version of cerebral activity as recorded from limited electrodes on the neonatal scalp, which is derived by filtering frequencies below $2 \mathrm{~Hz}$ and above $15 \mathrm{~Hz}$, semilogarithmic amplitude compression and time compression [5]. aEEG is a reliable and highly sensitive diagnostic tool that can be used in NICU especially when there is limited availability of conventional full array videoEEG (cEEG), which is considered the gold standard test for neuro-monitoring. Additional advantage of aEEG is the easy interpretation of recording by simple patternrecognition, which can be particularly useful to healthcare providers in intensive care units without neurological background or formal electrophysiology training.
In the current issue of Indian Pediatrics, Sharma, et al. [6] have published a prospective observational study to evaluate the diagnostic utility of aEEG in predicting the short term neurodevelopmental outcome in term neonates with encephalopathy regardless of the cause. Per the authors, the need for conducting this study is the dearth of data regarding the utility of aEEG in neonatal encephalopathies other than hypoxic ischemic encephalopathy (HIE). Although the sample size was relatively small with 58 subjects, the study commendably included with a fairly wide spectrum of etiologies for encephalopathy with HIE being the major cause followed by infection. The authors' decision to exclude neonates with major congenital malfor-mations, chromosomal abnormalities, neuronal migration disorders was reasonable, probably due to inherently high risk of global developmental delay and/or death in this subset of patients due to systemic, non-neurological issues. The definition of abnormal aEEG used in this study were compliant with the standard definitions of abnormal patterns reported in the routine aEEG monitoring. The aEEG correctly identified the encephalopathy (abnormal aEEG in $86 \%$ of the encephalopathic neonates). Similar to the prior studies mostly done in HIE subjects, this study demonstrated the statistically significant association between abnormal aEEG (abnormal background, immature or absent sleep-wake cycling and seizures) and the primary outcome measure (abnormal neurological exam at discharge and/or death) with a very high sensitivity at $100 \%$. The current study with its simple design elegantly corroborates and highlights the utility of aEEG in monitoring newborns to potentially enhance the neurological care and hopefully improve outcome.

Similar to the current study conducted by Sharma, et al. [6], there are several prior studies that corroborated the significance of aEEG as a predictive tool of neurological outcomes in neonatal encephalopathy, especially in neonates with hypoxic ischemic encephalopathy. Naqeeb, 
et al. [7] demonstrated a close relationship between the aEEG and subsequent neurodevelopmental outcome. In their study, $91 \%$ of neonates with a normal aEEG were normal on follow up at $18-24$ months of age, while $77 \%$ of infants with moderately abnormal or suppressed aEEG and/ or seizures died or developed neurologic abnormalities [7]. Osredkar, et al. [8] demonstrated that early onset of sleepwake cycling (within 36 hours) and a normal sleep wake cycle pattern in neonates with hypoxic ischemic encephalopathy were associated with a favorable Griffith developmental quotient between 1-5 years of age. A systematic review published by Rio, et al. [9] confirmed that aEEG back-ground activity during the first 72 hours of life has a strong prognostic value in infants with HIE, in terms of predicting neurological outcomes such as death or moderate/severe disability.

The current study adds more evidence on the utility of aEEG in all encephalopathic newborns. Despite this interesting result, it is worth mentioning that aEEG has an innate limitation due to the high occurrence of artifacts which can alter the interpretation, especially for seizures $[10,11]$. It is always useful to review the raw EEG data to confirm, given the high occurrence of seizures in this study (74\%). While the short term prognosis at discharge is very useful, it is also important to compare with long term data in infancy at 18-24 months where more sophisticated developmental testing could be performed, and these are no effect of hypothermia or sedative medications.

In summary, aEEG is a simple non-invasive monitoring tool that could provide important data regarding cerebral function during the critical postnatal course for newborns with encephalopathy. More studies are needed to evaluate the changes in aEEG trends with treatments and interventions during the NICU course in relation to the short and long term outcomes.

Funding: None; Competing interests: None stated.

\section{REFERENCES}

1. Antecedents of neonatal encephalopathy in the Vermont Oxford Network Encephalopathy Registry. Pediatrics. 2012;130:878-86.

2. Neonatal Encephalopathy and Neurologic Outcome, Second Edition.Pediatrics. 2014;133: e1482-488.

3. Aslam S, Strickland T, MolloyEJ. Neonatal encephalopathy: Need for recognition of multiple etiologies for optimal management. Front Pediatr. 2019;7:142.

4. Ahearne CE, Boylan GB, Murray DM. Short and long term prognosis in perinatal asphyxia: An update. World J Clin Pediatr. 2016;5:67-74.

5. Hellstrom-Westas L, Rosen I, de Vries LS, Grseisen G. Amplitude -integrated EEG classification and interpretation in preterm and term infants. NeoRev. 2006;7:e76-87.

6. Sharma GK, Natarajan CK, Hemanthkumar V, Sundaram S, Sharma SS. Prognostic value of amplitude integrated EEG in term neonates with encephalopathy. Indian Pediatr. 2021;58:928-31.

7. Naqeeb N, Edwards AD, Cowan FM, Azzopardi D. Assessment of neonatal encephalopathy by amplitudeintegrated electroencephalography. Pediatrics. 1999;103: 1263-271.

8. Osredkar D, Toet MC, van Rooij LGM, van Huffelen AC, Groenendaal F, de Vries LS. Sleep-wake cycling on amplitude integrated EEG in full-term newborns with hypoxic-ischemic encephalopathy. Pediatrics. 2005;115: 327-32.

9. Río RD, Ochoa C, Alarcon A, Arnáez J, Blanco D, GarcíaAlix A. Amplitude integrated electroencephalogram as a prognostic tool in neonates with hypoxic-ischemic encephalopathy: A systematic review. PLoS One. 2016; $1: 11: \mathrm{e} 0165744$.

10. Hagmann CF, Robertson NJ, Azzopardi D. Artifacts on the electroencephalogram may influence the amplitudeintergrated EEG classification: A qualitative analysis in neonatal encephalopathy. Pediatrics. 2006;118:2552-554.

11. Suk D, Krauss AN, Engel M, Perlman JM. Amplitudeintegrated electroencephalography in the NICU: frequent artifacts in premature infants may limit its utility as a monitoring device. Pediatrics. 2009;123:e328-32. 